\title{
Voronoi and Radical Tessellations of Packings of Spheres
}

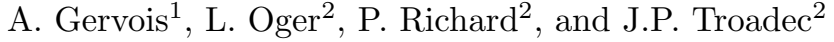 \\ 1 Service de Physique Théorique, Direction des Sciences de la Matière, \\ CEA/Saclay, F91191 Gif-sur Yvette Cedex \\ gervois@spht.saclay.cea.fr \\ 2 Groupe Matière Condensée et Matériaux, \\ UMR CNRS 6626, Université de Rennes 1, \\ Campus de Beaulieu, Bâtiment 11A, F35042 Rennes Cedex \\ \{Luc.Oger, Patrick.Richard, Jean-Paul.Troadec\}@univ-rennes1.fr
}

\begin{abstract}
The Voronoi tessellation is used to study the geometrical arrangement of disordered packings of equal spheres. The statistics of the characteristics of the cells are compared to those of $3 \mathrm{~d}$ natural foams. In the case of binary mixtures or polydisperse assemblies of spheres, the Voronoi tessellation is replaced by the radical tessellation. Important differences exist.
\end{abstract}

\section{Introduction}

An important element in the understanding of unconsolidated granular media is the description of the local arrangement of the grains and the possible correlations between them. The simplest model consists in monosize assemblies of spherical grains; the neighbourhood and steric environment of a grain can be described through the statistical properties of its Voronoi cell.

The Voronoi cells generate a partition of space (foam in three dimensions, mosaics in two dimensions) which may be considered without any reference to the underlying set of grains. Foams have been extensively studied in other contexts [1]. Some powerful experimental laws are known, at least for twodimensional systems. We used the Voronoi construction to study the local arrangement of $2 \mathrm{~d}$ disk assemblies [2] : results were qualitatively not too different from those observed on random classical mosaics but quantitative behaviour was much altered by steric constraints and the assembling procedure. We have performed the same study for equal spheres and verified that for our foams, the "universal" laws are again valid, again with quantitative steric constraints.

The next step consists in considering assemblies of unequal spheres. Several generalizations of the Voronoi construction were proposed, and their use depends on the problem under consideration. Though somewhat artificial, the radical tessellation $[3,4]$ keeps main topological features of the ordinary Voronoi tessellation and is well adapted to grain hindrance or segregation problems. 
For a physicist, the main interest consists in analysing the randomness of the packing or the transition between a fluid-like and a solid-like packing. So, in the present paper, most attention is paid to disordered packings. It is intended as a small review on our contribution to the geometrical analysis of packings of spheres with emphasis on two points : the interpretation of the tessellation as a random froth and the case of binary mixtures. We first recall some classical algorithms for building sphere assemblies (Sec.2), then give geometrical properties of the packings of spheres, both in the monosize (Sec.3) and the binary cases (Sec.4).

\section{Computer simulations}

The main parameter is the packing fraction $C$

$$
C=\frac{\text { volume of spheres }}{\text { total volume }}
$$

For a 3-dimensional packing of monosize grains, it runs from $C=0$ to $C=$ $\pi / 2 \sqrt{3}=0.7404$. which is realized in the face centered cubic (FCC) and hexagonal compact (HCP) ordered packings. The lower limit is obtained by a generation of points inside a 3D space (Poisson distribution [5] or Meijering model [6]). For all packing fractions between these two limits, a lot of techniques exist in order to generate a packing. We recall here algorithms which we used to get a packing of spheres as disordered as possible. It is possible now to consider large packings (15000 spheres) and so to get good statistics.

These algorithms generally hold both for monosize or polydisperse assemblies and of course, in the bi-dimensional case (disk assemblies). A detailed presentation may be found in [7]. They can be classified in two well-defined groups : the static and the dynamic methods of construction.

In the static case, the grains are placed at a given time step and cannot move afterwards. The extension of the Poisson process is the Random Sequential Adsorption model (RSA) where the particle is no longer a point [8]. This procedure does not provide very dense packings, the jamming limits for monosize particles being close to 0.38 for a three-dimensional packing of spheres and to 0.55 for a two-dimensional packing of disks. If there is a binary or a polydisperse distribution of size, the jamming limit can vary drastically according to the way the rejection is made. We also use the Modified Random Sequential Adsorption algorithm (MRSA) [9] which provides a slightly higher packing fraction, but introduces some anisotropy.

In models which generate dense disordered packings of disks or spheres the grains are placed according to a "gravity" [10,11] or a central field [12] and cannot move after this placement. These algorithms give a packing fraction close to 0.60 , generally smaller than the maximum possible for disordered systems $\left(C_{R C P} \approx 0.64\right)$. On the other hand, due to the building procedure, they clearly 
present some anisotropy.

The dynamic approach uses the initial position of the grains as an input parameter ; some short or long range interactions between grains generate displacements of the particles and reorganizations of the whole packing. The final positions of the particles and the possible values of the final packing fraction depend strongly on the process which can be collective or individual.

We specially used two algorithms :

- The Jodrey-Tory algorithm [13] and its modified version by Jullien et al [14], where any packing fraction value may be reached

- A Molecular Dynamics (event driven) code for granular media, specific of hard sphere systems [7] where all collisions are assumed to be instantaneous.

\section{Monosize packings}

\subsection{Topological quantities}

Distribution $\boldsymbol{p}(\boldsymbol{f})$ For any packing fraction $C$, the probability $p(f)$ to have a $f$-faceted cell is a single-peaked function with a maximum close to $f=15$ (Fig. 1), slightly asymmetric, with gaussian wings and it may be interpreted as the one which minimizes information at fixed averages $\langle f\rangle$ and $\left\langle f^{2}\right\rangle$ (the brackets $<$. $>$ stand for an average on face statistics). When the $C$ increases the distribution narrows rapidly and the dispersion $\mu_{2}$ decreases. The average number of faces $\left\langle f>\right.$ decreases too, from $2+48 \pi^{2} / 35=15.53 \ldots$ corresponding to the Random Poisson Voronoi (RVP) [5] or Meijering case [6]) to 14 (Fig. 2); notice that it is always larger than the maximum number (12) of contacts.

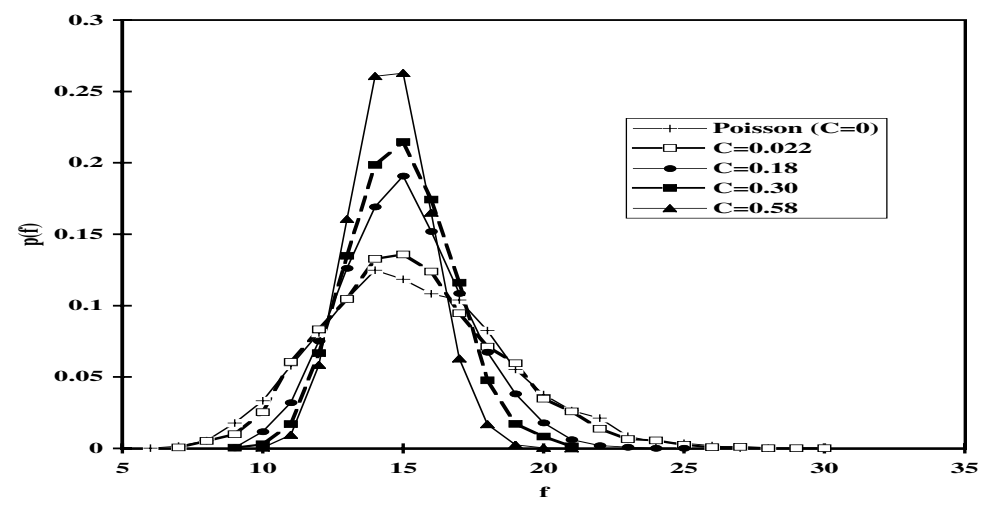

Fig. 1. Probability distribution $p(f)$ of $f$-faceted cell for different packing fractions $C$

These quantities depend not only on the packing fraction but also on the algorithm used, i.e. on the history of the packing: first, for different algorithms and the same packing fraction, $\langle f\rangle$ is larger for algorithms with some anisotropy, such as MRSA [9] or Visscher-Bolsterli [11] and Powell [10] algorithms where the 


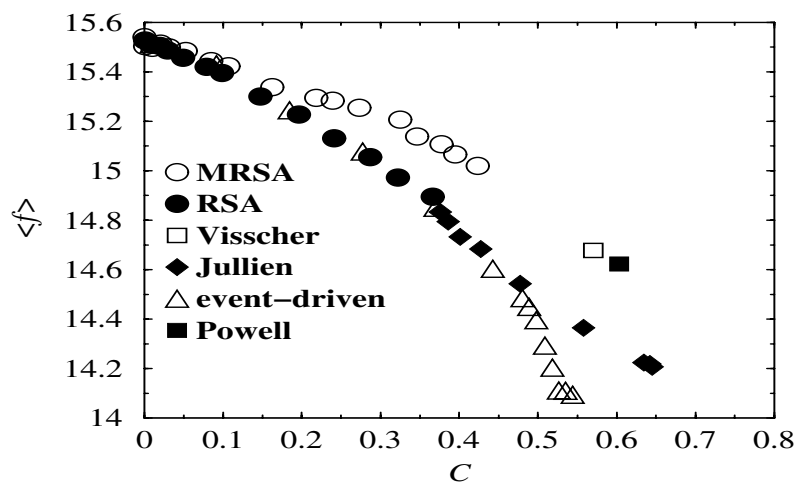

Fig. 2. Evolution of the mean number of faces versus the packing fraction, $C$, for different algorithms.

direction of gravity is favored. Secondly, for a given algorithm, it may be checked that cells become more isotropic when $C$ increases. The dependence of $f$ on anisotropy is in agreement with a theory developed by Rivier [15]. Let us point out the case of event-driven algorithm: for high packing fractions $(C>0.545)$ the system crystallizes [16]; the cells are then more isotropic than those of disordered packings at the same packing fraction and the limit value for $\langle f\rangle$ is 14 , i.e. the average number of neighbours (in the Voronoi sense) in slightly distorted FCC or HCP arrays [17].

So the packing fraction is clearly not a good quantity to describe the state of a foam and we look for a better parameter. On Fig. 3, we have plotted $<f>$ as a function of the sphericity coefficient $K_{s p h}$

$$
K_{s p h}=36 \pi\left\langle V^{2}\right\rangle /\left\langle A^{3}\right\rangle
$$

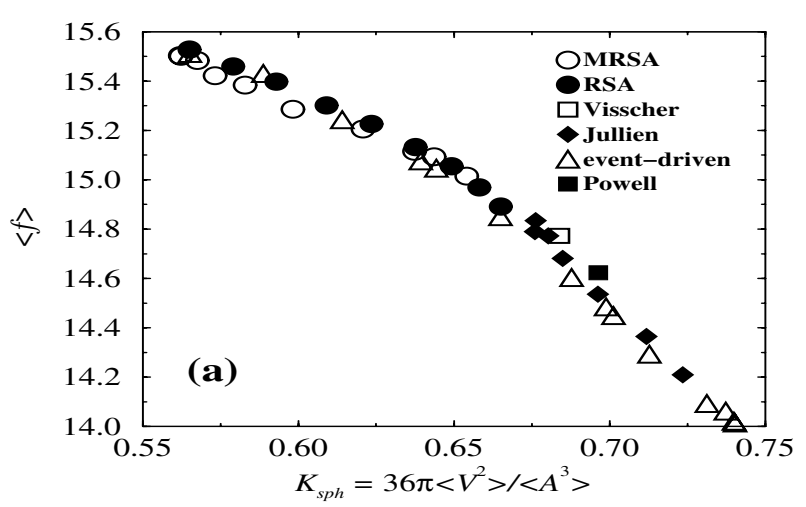

Fig. 3. Evolution of the mean number of faces versus the sphericity coefficient $K_{s p h}$ for all the algorithms used.

It turns out that points are positioned on a unique curve. Then, $K_{s p h}$ appears to be the relevant parameter [18]. 
Average number of edges For any $f$-faceted cell and any packing fraction, we have checked the relation $n(f)=6-12 / f$ and computed its average $\langle n(f)\rangle$. It is actually nearly constant on the whole packing fraction range $(<n(f)>\approx 5.17)$ because $\langle f>$ is a function slowly varying with $C$. As already noticed by Voloshin and Medvedev [19], most faces are 4, 5 and 6-sided (from $66 \%$ in the dilute case up to $80 \%$ in the most compact samples); however, 5 -sided faces are not really preeminent ( $40 \%$ in the regular lattices).

The distribution of the number of sides per face is a very adequate tool for studying the transition from a disordered to an ordered packing in dense systems as the thermalization goes on. We have plotted on Fig. 4 the fraction $p_{i}$ of faces with $i=4,5$ and 6 edges in event driven systems as the packing fraction increases. In the $C$ range where crystallization may occur, they behave quite differently in the stable branch (crystal) and the metastable one (supercooled liquid) [16].

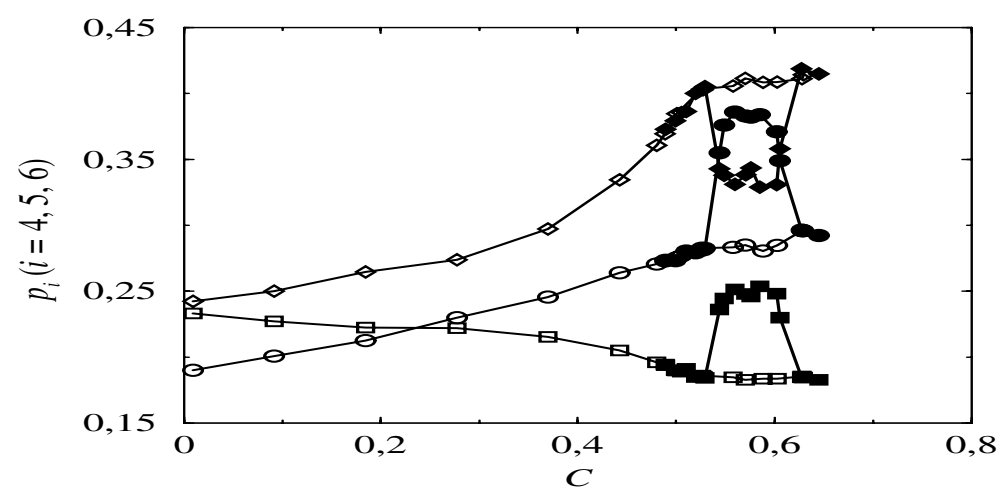

Fig. 4. Values of $p_{4}(\square) p_{5}(\diamond)$ and $p_{6}(\circ)$ for disordered packings (empty symbols) and for initially metastable packings (full symbol).

First neighbours The relations of a $f$-faceted cell with its neighbour cells is described by their average number of faces $m(f)$ and related quantities. The generalization in three dimensions of Weaire's identity [20] is easily checked.

$$
<f m(f)>=<f^{2}>
$$

Moreover, at any packing fraction, the average number of faces $f m(f)$ of all neighbours is a linear function of $f$,

$$
f m(f)=(<f>-a) f+a<f>+\mu_{2}
$$

which is precisely the Aboav-Weaire law [21] which has been shown to be exact in some particular $3 d$ systems [22]. Like $\left\langle f>\right.$ and $\mu_{2}$, the parameter $a$ depends on the packing fraction $C$.

For $2 \mathrm{~d}$ foams, Peshkin et al. [23] suggested that the linear dependence on the number of neighbours of Aboav's law was a consequence of the MAXENT 
principle applied to the topological correlation numbers. The corresponding correlation quantities in $3 \mathrm{~d}$ are the $A(f, g)$, related to the symmetric probability that two cells with $f$ and $g$ faces are neighbors. We measured them on several samples of 12000 objects both for Powell packings and RVP point systems. They are increasing functions of $f$ and $g$ (Figure 5), and although the precision is not very good, we can represent them by a linear dependence in $f$ and $g$ [24]

$$
A(f, g)=\sigma(g-<f>)(f-<f>)+f+g-<f>.
$$

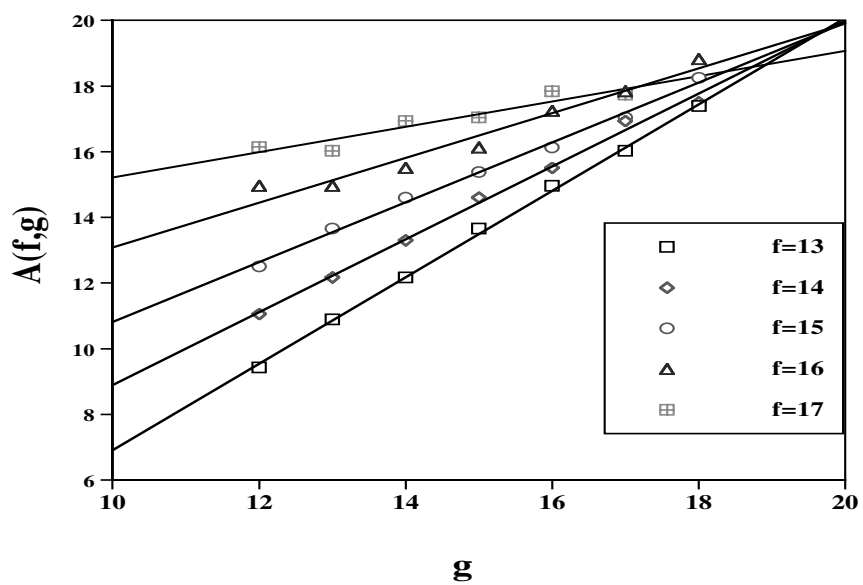

Fig. 5. Variation of $A(f, g)$ with $g$ for 12000 spheres for a Powell's algoritm (to be compared to its analog $A(n, m)$ for edges in 2d-mosaics [23])

\subsection{Metric properties}

We focus mainly on the volume of the cells, first as a function of the number of faces, then globally. For any packing fraction, the cell volume $V(f)$, is a linear function of $f$

$$
V(f)=V_{0}\left[1+(f-<f>) / K_{V}\right]
$$

where $V_{0}=\sum_{f} V(f) p(f)$ is the average volume, and $K_{V}$ is a parameter depending on the packing fraction $C$ (see Fig. 6). Linear dependence on $f$ exists also for the interfacial area and total length of edges. Thus, generalizations of Lewis [25] and Desch [26] laws are possible, even at high packing fractions, in opposition with the $2 \mathrm{~d}$ case, where steric exclusion implied the existence of a minimum at medium and high packing fractions. This is probably due to the fact that the number of faces is always high $(f \geq 10)$. The curves would probably not be linear any more if many cells had 6,7 or 8 faces, since polyhedra with a small number of faces require a larger volume than higher order ones [27].

Notice that, at the same packing fraction, the slope of the normalized volume is larger than that of the area or of the length. Thus, everything else being equal, the linear volume law (Lewis) is the most relevant and discriminatory. 


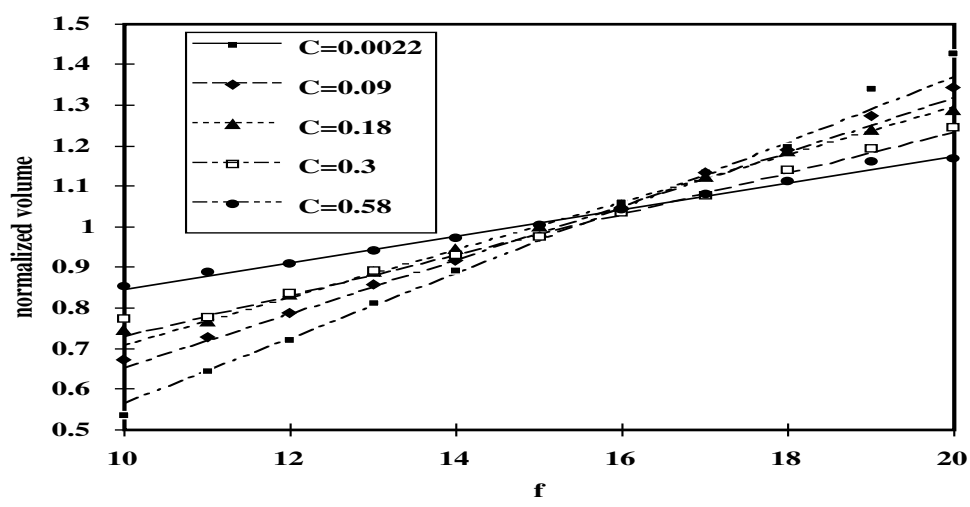

Fig. 6. Variations of the normalized volume of a $f$-faceted cell versus $f$ for different packing fractions $C$ (between 0.0022 and 0.58).

\section{Binary mixtures}

It is known that the arrangement of grains strongly depends on their geometry rather than on their physical properties, so that the relative size of a grain and their numerical proportion in the packing are the main relevant quantities. In binary mixtures, we have two species, with radii $R_{i}, i=1,2\left(R_{2}>R_{1}\right)$, and numerical proportions $n_{i}\left(n_{1}+n_{2}=1\right)$; we choose $R_{1} / R_{2}>\sqrt{3 / 2}-1=$ 0.224 . so that small grains cannot be trapped in the tetrahedron made with four contacting large spheres without any increase in volume.

\subsection{Radical tessellation}

The first thing consists in partitioning space by cells containing one grain. The Voronoi tessellation is no longer adequate: in dense packings, the cell can "cut" the larger grain and touching spheres may not be neighbors in the Voronoi sense. Several generalizations have been proposed, which all reduce to the usual tessellation in the monosize case and are generalized without difficulty to the polydisperse case. The simplest consists in the radical tessellation $[3,4]$, where the bisecting plane is replaced by the radical plane (all the points in the radical plane have the same tangency length - or power- for the two spheres). The definition may be somewhat artificial, but many of the features of the Voronoi tessellation are maintained: the cells are convex polyhedrons with planar faces, each one containing one grain, two touching spheres have a common face (the tangent plane) and thus may be considered as neighbors. The incidence properties still hold : a face is generically common to two cells, an edge to three and a vertex to four cells. Moreover, big grains have in general larger cells than smaller ones, a way again for estimating their relative hindrance.

\subsection{Topological and metric quantities}

It may be interesting to consider each species separately. Then we define partial quantities such as the partial distributions $p_{i}(f)$, partial average number of 
neighbor cells $\left\langle f_{i}>\right.$, average number of sides of a neighbor cell $m_{i}(f)$, partial average volume $\left\langle V_{i}(f)>\right.$, ... with obvious definitions ; we have

$$
<f>=n_{1}<f_{1}>+n_{2}<f_{2}>
$$

and similar relations for the other total averages. When the packing is not too dense, the relative size of the two kinds of grains is not very important and most properties of the monosize case remain more or less valid. When the packing fraction increases, the size of the cells begins to depend strongly on the size of the grains: small grains have smaller cells than large grains and consequently less neighbors and the two populations can be very separated as can be seen for a size ratio equal to 2 for the distributions $p_{i}(f)$ and $p(f)$ (Fig. 7) and the volume distribution (Fig.8) respectively.

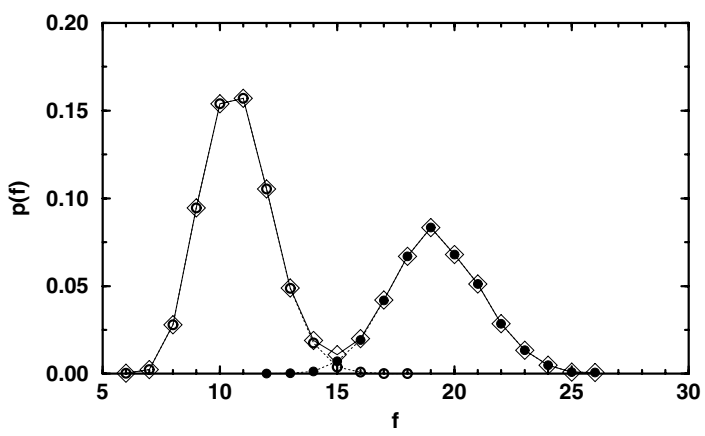

Fig. 7. Distribution $p(f)(\diamond)$ of the number of cell sides and weighted partial distributions $n_{i} p_{i}(f)(\circ: i=1, \bullet: i=2)$ for $k=2$ and $n_{1}=0.6$ for $C=0.61$

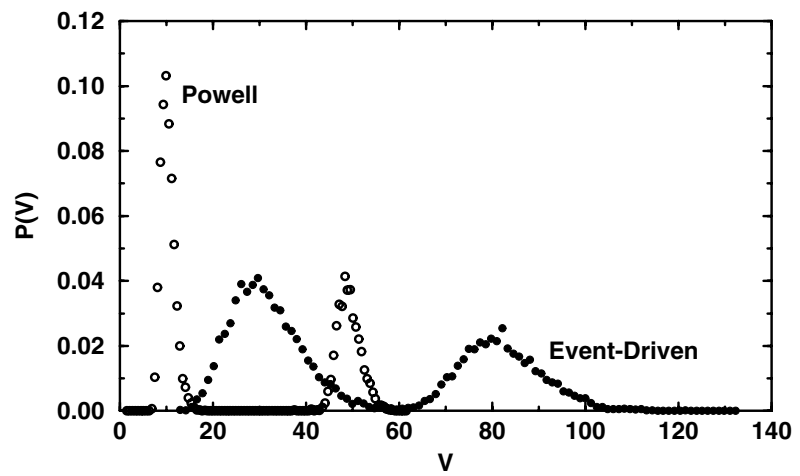

Fig. 8. Volume distribution $\mathrm{P}(\mathrm{V})$ at $C=0.31$ (Event-Driven) and $C=0.61$ (Powell) for $n_{1}=0.6$. The length unit is the value of the small sphere radius

We shall not insist very much on these results. Most of them may be found in [28]. Let us just list main features, assuming $R_{2}>R_{1}$ : 
- distribution $p(f)$ is a one peaked function at low packing fractions, and separate into two distinct distributions as $C$ increases. Then, $<f_{1}>\leq<f_{2}>$ and the total average coordination number may be smaller than 14 .

- separately each species obeys Aboav-Weaire law, with different slopes. The whole packing does not obey Aboav law and the curve is S-shaped.

- partial volumes $V_{i}(f)$ and areas $A_{i}(f)$ do not verify Lewis nor Desch law and of course, the total averages do not either. The volume (resp. area) distribution is a two peaked-function.

The same behaviour is probably true for ternary mixtures, with possibly 3 peaks. When the number of species involved increases, a smoothing may appear, depending on the radii size distribution and polydisperse assemblies may provide an intermediate behaviour.

\section{Conclusion}

We have given here a description of the geometrical organization of disordered packings of spheres, analyzed through the statistical properties of their Voronoi (and radical) cells. In the case of equal spheres, the Voronoi tessellation behaves like an ordinary foam and follows classical universal laws (Aboav, Lewis,...) which are known from a long time in two dimensions and which we find to be true in $3 \mathrm{~d}$ either ; numerical values depend on the packing fraction and the steric constraints due to the grain hindrance. Differences between disordered and ordered packings may be seen easily on very simple quantities like the distribution of the number of edges of the faces.

In the case of binary mixtures, the two species behave very differently at high density and the empirical laws for ordinary foams do not hold. On the other hand, radical tessellation is a good tool to test the possible segregation in the repartition of the two species [29]. For polydisperse assemblies, an intermediate behaviour is expected.

\section{References}

1. see for instance Weaire D., Rivier N., : Soap, Cells and Statistics - Random Patterns in two Dimensions, Contemp. Phys. 25 (1984) 59

2. Lemaitre J., Gervois A., Troadec J.-P., Rivier N., Ammi M., Oger L. and Bideau D.: Arrangement of cells in Voronoi tessellations of monosize packings of discs, Phil. Mag. B, 67 (1993) 347

3. Gellatly B.J. and Finney J.L.: Characterization of models of multicomponent amorphous metals : the radical alternative to the Voronoi polyhedron, J. Non Crystalline Solids 50 (1982) 313

4. Telley H., Liebling, T.M., and Mocellin A.: The Laguerre model of grain growth in two dimensions, Phil. Mag. B73 (1996) 395-408

5. Okabe A., Boots B., Sugihara K. and Chiu S.N., in Spatial Tessellations : concepts and applications of Voronoi diagrams, Wiley (2000)

6. Meijering J.L.: Interface area, edge, length and number of vertices in crystal aggregates with random nucleation, Philips Res. Rep., 8 (1953) 270 
7. Oger L., Troadec J.-P., Gervois A. and Medvedev N.N.: Computer simulations and tessellations of granular Materials, in Foams and Emulsions J.F. Sadoc and N. Rivier eds, Kluwer (1999), p527

8. Feder J.: Random Sequential Adsorption, J. Theor. Biol. 87 (1980) 237

9. Jullien R. and Meakin P.: Random sequential adsorption with restructuring in two dimensions, Journal of Physics A: Math. Gen., 25 (1982) L189-L194

10. Powell M.J.: Site percolation in randomly packed spheres, Phys. Rev. B 20 (1979) 4194

11. Visscher W.H. and Bolsterli H.: Random packing of equal and unequal spheres in two and three dimensions, Nature, 239 (1972) 504

12. Bennett C.H.: Serially deposited amorphous aggregates of hard spheres, J. Appl. Phys., 43 (1972) 2727

13. Jodrey W.S. and Tory E.M.: Computer simulation of random packings of equal spheres, Phys. Rev. A, 32 (1985) 2347

14. Jullien R., Jund P., Caprion D. and Quitman D.: Computer investigation of long range correlations and local order in random packings of spheres, Phys. Rev. E, 54 (1996) 6035

15. Rivier N.: Recent results on the ideal structure of glasses, Journal de Physique Colloque C9 (1982) 91-95

16. Richard P., Oger L., Troadec J.-P. and Gervois A.: Geometrical characterization of hard sphere systems, Phys. Rev. E 60 (1999) 4551

17. Troadec J.-P., Gervois A. and Oger L.: Statistics of Voronoi cells of slightly perturbed FCC and HCP close packed lattices, Europhys. Lett. 42 (1998) 167

18. Richard P., Troadec J.-P., Oger L. and Gervois A.: Effect of the anisotropy of the cells on the topological properties of two- and three-dimensional froths, Phys. Rev. E 63 (2001) 062401

19. Voloshin V.P., Medvedev N.N. and Naberukin Yu. I.: Irreguler packing Voronoi Polyhedra I \& II, Journal of Structural Chemistry, 26 (1985) 369 \& 376

20. Weaire D.: Some remarks on the arrangement of grains in a polycrystal, Metallography, 7 (1974) 157

21. Aboav D.A.: The Arrangement of cells in a Net, Metallography, 13 (1980) 43

22. Fortes M.A.: Topological properties of cellular structures based on staggered packing of prisms, J. Phys. France, 50 (1989) 725,

23. Peshkin M.A., Strandburg K.J. and Rivier N.: Entropic prediction for cellular networks, Phys. Rev. Lett., 67 (1991) 1803

24. Oger L., Gervois A., Troadec J.-P. and Rivier N.: Voronoi tessellation of spheres : topological correlations and statistics, Phil. Mag. B 74 (1996) 177-197

25. Lewis F.T.: The correlation between cell division and the shapes and sizes of prismatic cells in the epidermis of cucumis, Anat. Record., 38 (1928) 341

26. Desch C.H.: The solidification of metals from the liquid state, J. Inst. Metals, 22 (1919) 24

27. Fortes M.A.: Applicability of the Lewis and Aboav-Weaire laws to 2D and 3D cellular structures based on Poisson partitions, J. Phys. A 28 (1995) 1055

28. Richard P., Oger L., Troadec J.-P. and Gervois A.: Tessellation of binary assemblies of spheres, Physica A 259 (1998) 205

29. Richard P., Oger L., Troadec J.P. and Gervois A.: A model of binary assemblies of spheres, EPJE (2001) in press 